\title{
An sEMG-Based Method to Adaptively Reject the Effect of Contraction on Spectral Analysis for Fatigue Tracking
}

\author{
Kaan Gokcesu \\ EECS, Massachusetts Institute \\ of Technology \\ Cambridge, Massachusetts, \\ United States, \\ gokcesu@mit.edu
}

\author{
Mert Ergeneci \\ Hercules Biomedical R\&D, \\ Ankara, Turkey \\ ergenecimert@gmail.com
}

\author{
Erhan Ertan \\ Hercules Biomedical R\&D, \\ Ankara, Turkey \\ erhertan@gmail.com
}

\author{
Abdallah Zaid Alkilani \\ Bilkent University, \\ Ankara, Turkey \\ abdallah@ug.bilkent.edu.tr
}

\author{
Panagiotis Kosmas \\ School of Natural and \\ Mathematical Sciences, King's \\ College London, \\ London, United Kingdom \\ panagiotis.kosmas@kcl.ac.uk
}

\begin{abstract}
Muscle fatigue detection and tracking has gained significant attention as the sports science and rehabilitation technologies developed. It is known that muscle fatigue can be evaluated through surface Electromyography (sEMG) sensors, which are portable, non-invasive and applicable for real-time systems. There are plenty of fatigue tracking algorithms, many of which uses frequency, time and time-frequency behaviors of sEMG signals. An example to most commonly used sEMG-based fatigue detection methods can be mean frequency (MNF), median frequency (MDF), zero-crossing rate (ZCR) and continuous wavelet transform (CWT). However, all of these muscle fatigue calculation methods are adversely affected by the dynamically changing sEMG contraction amplitude, since EMG spectrum also demonstrates a shift with the changing signal RMS; powerful contractions lead a shift to high frequency bounds and the opposite happens for the weak. To overcome that, we propose an adaptive algorithm, which learns the effect of contraction power on sEMG power spectral density (PSD) and subtracts that amount of frequency shift from the PSD.
\end{abstract}

\section{ACM Classification Keywords}

H.5.m. Information Interfaces and Presentation (e.g. HCI): Miscellaneous

\section{Author Keywords}

sEMG Signal Processing; Muscle Fatigue Tracking; Spectral Analysis, Wearable Computing

Permission to make digital or hard copies of all or part of this work for personal or classroom use is granted without fee provided that copies are not made or distributed for profit or commercial advantage and that copies bear this notice and the full citation on the first page. Copyrights for components of this work owned by others than the author(s) must be honored. Abstracting with credit is permitted. To copy otherwise, or republish, to post on servers or to redistribute to lists, requires prior specific permission and/or a fee. Request permissions from permissions@acm.org.

ISWC '18, October 8-12, 2018, Singapore, Singapore

(C) 2018 Copyright held by the owner/author(s). Publication rights licensed to ACM ISBN 978-1-4503-5967-2/18/10 . \$ \$15.00

DOI: https : //doi .org/10.1145/3267242 . 3267292

\section{INTRODUCTION}

Muscle fatigue is the inability of the muscle to generate the desired contraction and force, as a result of repetitive and powerrequiring tasks undergone [9]. The detection and tracking of muscle fatigue has gained importance in the sports science and rehabilitation applications, since it can lead to prevent muscle injury, over-training and re-injury during rehabilitation; analyze muscle strength and endurance development; and monitor gradual athlete performance [5, 12]. Captured sEMG signals provide information about muscle fatigue. An sEMG signal can be evaluated in both time and frequency domains in order to investigate muscle fatigue. Physiologically, a simple muscle contraction is actualized by the impulses (i.e., firings) sent from brain to the motor unit of the muscle. The amplitude of the impulses is fixed and does not affect the magnitude of contractions. The contractions vary through the rate of impulses, i.e. the firing rate [2]. When a muscle begins undergoing fatigue, substances such as $\mathrm{H}^{+}, \mathrm{Ca}^{+2}, \mathrm{Na}^{+}$, and $\mathrm{P}^{-3}$ accumulate and lessen the conduction velocity in muscle fibers and motor units [8]. The reduced conduction velocity results in a direct decrease in the impulse rate, prompting the muscle's contraction to fall below the desired amount. This constitutes the definitions of fatigue. Nevertheless, the decrease of the firing rate does not only reduce the contraction amount, which is mainly observable through the change in the RMS value; the decrease of the firing rate also shifts the spectral density to smaller frequency values.

Frequency, time, and frequency-time domain analysis can be conducted to trace muscle fatigue. In frequency-domain analysis, Mean Frequency (MNF) and Median Frequency (MDF) dominate as the current state of the art $[7,11]$. As for timedomain analysis, Zero Crossing Rate (ZCR) is amongst the relevant analysis methods nowadays [3]. Lastly, frequency-time domain analysis incorporates both time-domain (i.e. RMS) and frequency-domain (i.e. $\mathrm{MNF} / \mathrm{MDF}$ ) features as its parame- 
ters, Continuous Wavelet Transform (CWT) being an example of such analysis methods $[13,15]$. For EMG fatigue tracing, the choice of method depends on the analysis approach. For instance, $\mathrm{MNF} / \mathrm{MDF}$ are more preferable for online short-term fatigue tracking, CWT for long-term big-batch analysis [16]. Nevertheless, EMG amplitude fluctuations affect the robustness of any method chosen for fatigue analysis [10]. To be specific, contraction amplitude fluctuations have an adverse effect on the fatigue analysis of dynamic exercises, where irregular and varying contractions magnitudes are actualized. For example, when a small-amplitude contraction is followed by a high-amplitude contraction, the power spectrum would invariably shift to lower frequencies. However, as discussed earlier, this may result in potential muscle fatigue misdiagnosis, prompting the need for a tool that dismisses the effects of contraction amplitudes on fatigue analysis.

Thus, we have developed an adaptive algorithm that suppresses the effects of contraction amplitude (i.e., Contraction Effect Suppression, CES) on fatigue analysis. It is worth mentioning that we are not proposing a method, but a correctional tool that can aid preexisting methods (cf. MNF/MDF, ZCR, CWT) in rejecting the effects of contraction amplitude fluctuations. Since for a powerful contraction the neural system is required to send impulses to the motor units of the muscles with a firing rate that is substantially higher than that of a weak contraction. In particular, it can be said that the firing rate, besides the muscle fatigue, is also related to the amount of contraction power, resulting a frequency shift to higher bounds with powerful contractions and the opposite with weak contractions. The proposed tool, CES, relies on the linear relation between RMS and spectral shift, which are universal features of EMG signals, and is therefore compatible with any choice of fatigue analysis method. The presented method, simply learns the amount of spectral shift that the sEMG RMS adds to the system and subtracts it from the PSD as an offset.

\section{PROBLEM SETUP}

In this paper, we are dealing with the problem of fatigue estimation from sEMG signals during varied physical activities that involve gradually changing signal amplitude. Since changes in EMG signal amplitude affect the Power Spectral Density (PSD), fatigue tracking through spectral analysis such as Median Frequency (MDF) and Mean Frequency (MNF) are singly inefficient at detecting fatigue during dynamic activities. In order to reduce the effect of signal amplitude (i.e. Root Mean Square), we have proposed a mathematical model of an observed EMG frame's spectral behavior. Assume that we use a generic metric $\kappa$ to track fatigue level. $\kappa$ can be chosen as any analysis metrics (i.e. MNF, MDF, or ZCR ) for tracking the localized fatigue level. Then, the proposed mathematical model assumes a linear relation between the contraction amplitude and the time-frequency analysis, as

$$
\kappa_{n}=\theta_{n} * r_{n}+\frac{1}{f_{n}},
$$

where $\kappa_{n}$ is the fatigue tracking metric, $r_{n}$ is the Root Mean Square (RMS) value and $f_{n}$ is the clean fatigue level of the $n^{t h}$ frame. If the $\theta_{n}$ value is estimated, then the clean fatigue value $f_{n}$ can be extracted using (1). However, the fatigue level $f_{n}$ is not negligible since it also changes with gradual muscle activity and directly affects the $\kappa$ value. Thus, in order to neglect the effect of changes in $f_{n}$, we consider contraction frames realized with very small time differences. Since the change in $f_{n}$ converges to zero as the time difference between two contraction windows is minimized, we ignore the impact of fatigue on spectral analysis and estimate the weight, $\widehat{\theta}$, as

$$
\widehat{\boldsymbol{\theta}}=\left(\sum_{i, j}^{N} \kappa_{i}-\kappa_{j}\right) /\left(\sum_{i, j}^{N} r_{i}-r_{j}\right), \forall i \neq j,
$$

where $i$ is the number of the last (for batch analysis) or current ( for online analysis) frame and $j$ is the number of all the other frames except the $i$. Nonetheless, the computation of $\widehat{\theta}$ in (2) can be misleading since the RMS and $\kappa$ differences are calculated with uniform weighting, which is incorrect due to the time-dependent change in the fatigue level. Thus, both differences must be modified by multiplying with a weight that is inversely proportional to the time difference between the contraction frames. We modified (2) using negative exponential weighting of the time differences between the frames as follows:

$$
\widehat{\theta}=\left(\sum_{i, j}^{N} e^{-\gamma\left(t_{i}-t_{j}\right)}\left(\kappa_{i}-\kappa_{j}\right)\right) /\left(\sum_{i, j}^{N} e^{-\gamma\left(t_{i}-t_{j}\right)}\left(r_{i}-r_{j}\right)\right)=\frac{K}{R},
$$

where $\gamma$ is the learning rate that is selected to be, $1 / \sqrt{t_{i}}$, decreasing over time. To track our fatigue observation $\kappa$ and the RMS of each contraction, we have created the EMG data frames using a sliding window. Let $N$ be the frame length and $L$ be the frame overlap length (such that two adjacent frames have a total of $L$ samples in common). We define the column vector $y_{t}$ as the frame at time $t$, given by:

$$
y_{t}=[y[m+1], y[m+2], \ldots, y[m+N]]^{T},
$$

where $m=(t-1)(N-L)$. The $y_{t}$ is defined as the superposition of clean EMG signal, $x_{t}$ and Additive White Gaussian Noise (AWGN), $v_{t}$ as the frames at time $t$. We also define the fourier transforms (DFT) of these column vectors as $Y_{t}, X_{t}, V_{t}$ respectively. Hence,

$$
\begin{gathered}
y_{t}=x_{t}+v_{t}, \\
Y_{t}=X_{t}+V_{t} .
\end{gathered}
$$

By using $Y_{t}$, we are going to calculate the $\kappa_{t}$ and $r_{t}$ in (1) at time $t$. In order to make such calculations, we use the frames that involve muscle activation potentials, namely sEMG signal. However, there is no guarantee that the muscle signals will be present at each observation frame (there may be frames where the muscle is not contracted). Hence, if there is no contraction at time $t$, then the observation frame will simply be given by $Y_{t}=V_{t}$. Therefore, we can write the model as:

$$
Y_{t}=\alpha_{t} S_{t}+V_{t},
$$

where $S_{t}$ is the muscle contraction signal, $X_{t}=\alpha_{t} S_{t}$, and $\alpha_{t}=1$ if there is a contraction and 0 otherwise. For these reasons, we first detect the EMG data frames where the muscle activation 
potential is present and use their information to estimate the fatigue level of the muscle.

\section{ACTIVE FRAME CLASSIFICATION}

To correctly detect the EMG data frames that contain muscle contractions, we need to use an unsupervised learning procedure since, in most prominent EMG applications, the corresponding time of the muscle contractions is not necessarily provided [4]. To decide whether an incoming data frame is active or not (i.e. contains contractions or not), we use the energy of that particular frame. At each time $t, P_{t}$ denotes the energy of the received frame $y_{t}$, which is given by: $P_{t}=y_{t}^{T} y_{t}$, where $y_{t}^{T}$ is the transpose of $y_{t}$. To decide whether an incoming frame $y_{t}$ is active or not, we compare a monotonically increasing transformation $\Phi\left(P_{t}\right)$ with a threshold $\tau_{t}$ :

$$
\alpha_{t}= \begin{cases}1, & \Phi\left(P_{t}\right) \geq \tau_{t} \text { (active) } \\ 0, & \Phi\left(P_{t}\right)<\tau_{t} \text { (inactive) }\end{cases}
$$

For the algorithm to function adequately, a suitable selection of the threshold $\tau_{t}$ is needed. However, without the knowledge of the energy levels for the active and inactive frames, this threshold cannot be reliably selected. Therefore, instead of selecting a fixed threshold, we use a dynamic thresholding scheme that learns with Online Gradient Descent (OGD) [6]. In general, OGD procedure updates the threshold as follows:

$$
\tau_{t+1}=\tau_{t}-\mu_{t} \frac{\partial l_{t}\left(\tau_{t}\right)}{\partial \tau_{t}}
$$

where $l_{t}(\cdot)$ is the loss function at time $t$. For the detection of maximal contractions, we need a threshold value closer to the maximum received frame energy. Therefore, we use the positive exponential transform on the energy values $P_{t}$, given by $\Phi\left(P_{t}\right)=\exp \left(P_{t}\right)$. The loss function consequently becomes:

$$
l_{t}\left(\tau_{t}\right)=\left(\tau_{t}-\exp \left(P_{t}\right)\right)^{2},
$$

which modifies the MMSE solution to:

$$
\tau^{*}=\underset{\tau}{\arg \min } \sum_{t=1}^{T}\left(\tau-\exp \left(P_{t}\right)\right)^{2}=\frac{1}{T} \sum_{t=1}^{T} \exp \left(P_{t}\right) .
$$

We would like to point out that the original MMSE solution is equivalent to the logarithm of the geometric mean of the exponentiated energy values $\exp \left(P_{t}\right)$. Hence, this $\tau^{*}$ is a satisfactorily higher threshold value due to the AM-GM inequality. Using the loss function in (10) and the OGD method in (9) with the step size $\mu_{t}=1 / 2 t$, our threshold update becomes:

$$
\tau_{t+1}=\tau_{t}-\frac{\tau_{t}-\exp \left(P_{t}\right)}{t},
$$

and our active frame detection is given by:

$$
\alpha_{t}= \begin{cases}1, & \exp \left(P_{t}\right) \geq \tau_{t} \text { (active) }, \\ 0, & \exp \left(P_{t}\right)<\tau_{t} \text { (inactive) } .\end{cases}
$$

We point out that this recursive approach is similar to the softmax operation. If, instead of $\exp \left(P_{t}\right)$, we use $\exp \left(K P_{t}\right)$ and let $K$ go to $\infty$ (i.e. instead of a unit gain we use infinite gain), the recursive operation will converge to the maximum value of the energies. Thus, after detecting the frames that are active (i.e. contractions), we begin estimating the clean fatigue level of localized muscles.

\section{CONTRACTION EFFECT SUPPRESSION (CES)}

In this Section, we propose the method of extracting the clean fatigue value $f_{n}$ in (1). This can be done by solely estimating and updating the weight of $\theta$ for each frame, since RMS is the amplitude indicator and $\kappa$ is any of the time-frequency domain fatigue tracking methods. The most common and frequently used $\kappa$ metrics in relevant literature are MNF, MDF, and ZCR, which are sequentially described in the following subsection.

\section{Preliminaries}

Prior to the estimation of $\theta$, it is first required to know the certain time-frequency domain fatigue tracking calculation methods (i.e. $\kappa$ ). MNF, MDF, and ZCR are a few examples of the methods frequently used to detect the spectral shift of sEMG signals, with which fatigue behavior is obtained. Since we have defined the mathematical model to extract the clean fatigue level in (1), RMS is also required besides the $\kappa$ metrics for proper estimation of $\theta$. Let $Y_{n}[f]$ be the Discrete Fourier Transform (DFT) of the received contraction frame $y_{n}$. In order to obtain sharper results, we used the Spectral Power $I_{n}[f]$ which is calculated as follows:

$$
I_{n}[f]=\underbrace{\left(Y_{n}[f] \circ Y_{n}[f]\right)}_{\text {Hadamard product }},
$$

where $I_{n}, y_{n}$ and $Y_{n}$ are column vectors.

Mean Frequency (MNF)

MNF, $m_{n}$, of the $n^{\text {th }}$ contraction frame is calculated as

$$
m_{n}=\frac{I_{n} F}{I_{n} I_{n}^{T}}
$$

where $I_{n}^{T}$ corresponds to the transpose of the PSD and $F$ is a column vector involving the frequency bins starting from 1 to half of the sampling frequency $(F s / 2)$.

\section{Median Frequency (MDF)}

In order to estimate the MDF of the $n^{\text {th }}$ frame, denoted $m d_{n}$, the cumulative sum $\mathrm{Cum}_{n}$ of the $I_{n}[f]$ is taken. Then, the halfsum of the elements of the Spectral Density $I_{n}[f]$ is calculated as $\mathrm{SumI}_{n}$. After calculating $\mathrm{SumI}_{n}$ and $\mathrm{Cum}_{n}$, the first index of $\mathrm{Cum}_{n}$ that is greater in value than $\mathrm{SumI}_{n}$ corresponds to the desired $m d_{n}$.

\section{Zero Crossing Rate (ZCR)}

The ZCR, denoted $z c r_{n}$, of the $n^{\text {th }}$ frame is determined by the amount of sign changes throughout the signal, which is formulated as:

$$
z c r_{n}=\frac{1}{N-1} \sum_{i=1}^{N-1} \mathbf{1}\left(y_{n}[i] y_{n}[i-1]<0\right),
$$

where $N$ is the frame length, $\mathbf{1}($.$) is the indicator function that$ returns 1 if the statement inside is true, or 0 otherwise.

Root Mean Square (RMS)

RMS of the $n^{\text {th }}$ contraction frame $r_{n}$ is calculated as

$$
r_{n}=\sqrt{\frac{y_{n} y_{n}^{T}}{N}}
$$


where $N$ is the frame length and $y_{n}^{T}$ is the transpose of the contraction frame $y_{n}$.

\section{Estimation of $\theta$}

It is known that the muscle contraction rate demonstrates a certain shift in spectral behavior of the sEMG signal, even when there is no change in the fatigue level of the subject. Regardless of the metric used to detect fatigue, the effect of contraction amplitude on the spectral behavior of the sEMG signal can be rejected through the estimation of $\theta$ in (1). In (3) of Section 2, we estimate the $\theta$ value by dividing the exponentially weighted (and inversely proportional with the time difference of the frames) sum differences of the fatigue metrics (i.e. $\kappa_{i}-\kappa_{j}, \forall i \neq j$ ) by RMS values (i.e. $r_{i}-r_{j}, \forall$ $i \neq j)$. The stated estimation of $\theta, \widehat{\theta}$, is shown as $\widehat{\theta}=K_{n} / R_{n}$, where $K_{n}$ and $R_{n}$ are the accumulated differences of $\kappa$ and $r$. The calculations of $K_{n}$ and $R_{n}$ are shown in (18) and (19).

$$
\begin{aligned}
& K_{n}=K_{n-1}+\sum_{i=1}^{n-1} e^{-\gamma(n-i)}\left(\kappa_{n}-\kappa_{i}\right) \\
& R_{n}=R_{n-1}+\sum_{i=1}^{n-1} e^{-\gamma(n-i)}\left(r_{n}-r_{i}\right)
\end{aligned}
$$

However, (18) and (19) are not feasible when there is a large amount of contraction data. Since the number of iterations is directly related to the total amount of contractions, the calculations of $K_{n}$ and $R_{n}$ will have increasing computational costs. However, $K_{n}$ and $R_{n}$ are efficiently calculable as

$$
\begin{gathered}
K_{n}=K_{n-1}+e^{-\gamma n}(\kappa_{n} \underbrace{\sum_{i=1}^{n-1} e^{\gamma i}}_{\zeta_{n-1}}-\underbrace{\sum_{i=1}^{n-1} e^{\gamma i} \kappa_{i}}_{\beta_{n-1}}), \\
R_{n}=R_{n-1}+e^{-\gamma n}(r_{n} \underbrace{\sum_{i=1}^{n-1} e^{\gamma i} r_{i}}_{\zeta_{n-1}^{n-1} e^{\gamma i}}),
\end{gathered}
$$

where $\zeta, \beta$ and $\delta$ are intermediate variables. Thus,

$$
\begin{aligned}
& K_{n}=K_{n-1}+e^{-\gamma n}\left(\kappa_{n} \zeta_{n-1}-\beta_{n-1}\right), \\
& R_{n}=R_{n-1}+e^{-\gamma n}\left(r_{n} \zeta_{n-1}-\delta_{n-1}\right) .
\end{aligned}
$$

We update $\zeta_{n}, \beta_{n}$ and $\delta_{n}$ as

$$
\begin{aligned}
& \zeta_{n}=\zeta_{n-1}+e^{-\gamma_{n}}, \\
& \beta_{n}=\beta_{n-1}+\kappa_{n} e^{-\gamma_{n}}, \\
& \delta_{n}=\delta_{n-1}+r_{n} e^{-\gamma_{n}} .
\end{aligned}
$$

After the calculation of $K$ and $R$ values with a single iteration for each new contraction frame, $\widehat{\theta}$ can be estimated as $K_{n} / R_{n}$. Through the use of $\widehat{\theta}$, the effect of amplitude on the spectral analysis is rejected, which is explained next.

\section{Clean Fatigue Extraction}

The clean fatigue level $f_{n}$ of the $n^{\text {th }}$ frame is calculated as the subtraction of $\theta_{n} r_{n}$ from the generic fatigue tracking metric $\kappa_{n}$. As stated, $\kappa$ is a generic fatigue tracking tool and can

\begin{tabular}{||c||c|c|c|c||}
\hline Subject No & Age & Gender & Height (cm) & Weight (kg) \\
\hline \hline S1 & 20 & Female & 167 & 61 \\
\hline S2 & 24 & Female & 169 & 67 \\
\hline S3 & 26 & Female & 165 & 58 \\
\hline S4 & 24 & Male & 173 & 72 \\
\hline S5 & 25 & Male & 178 & 68 \\
\hline S6 & 28 & Male & 168 & 70 \\
\hline S7 & 24 & Male & 167 & 72 \\
\hline S8 & 25 & Male & 189 & 66 \\
\hline S9 & 24 & Male & 175 & 78 \\
\hline S10 & 24 & Female & 181 & 60 \\
\hline
\end{tabular}

Table 1. Information about gender, age, height and weight of the subjects

be selected from the methods described in Section 4.1. The extraction of the clean fatigue metric is realized as

$$
f_{n}=\frac{1}{\kappa_{n}-\widehat{\theta}_{n} r_{n}} .
$$

\section{EXPERIMENTS}

In this section, we validate the performance of our method through several experiments. In the following subsection (Section 5.1), we detail the experimental setup (i.e. the number of subjects, the specifications of the sEMG sensor, the type of the physical activity and the sensor placement locations of data acquisition). Subsequently, in Section 5.2, the achievement of our proposed CES algorithm is validated with the use of various $\kappa$ selections. As the $\kappa, \mathrm{MDF}, \mathrm{MNF}$ and ZCR is chosen. Also, the window size is determined to be 128 with an overlap of 64. Finally, in Section 5.4 and 5.3, the extracted fatigue values with the use of CES are compared with the lactate and isokinetic dynamometer results for aerobic and anaerobic activities respectively.

\section{Experimental Setup}

In our experiments, sEMG data is gathered from 10 healthy subjects with various physical properties (i.e. age, gender, height $(\mathrm{cm})$ and weight $(\mathrm{kg}))$, with the details in Table 1 . The sensor used for EMG data acquisition is Biometrics $s \times 230-1000$, which is a dry active surface electromyography sensor with a pass band of $20-500 \mathrm{~Hz}$ and a gain of 1000 [1]. We have considered two different scenarios for the experimental data acquisition, which involved anaerobic and aerobic exercises. For the first scenario subjects performed an anaerobic exercise involving several sets consecutive biceps curls. As for an aerobic exercise, subjects made sets running and walking. Throughout the experiments, the sEMG sensor was placed on the most prominent bulge of the Gastrocnemius Medialis muscle for the aerobic, and the line between the Medial Acromion and the Cubital Fossa, at 1/3 of the distance starting from the Cubital Fossa, for the anaerobic exercise [14]. 

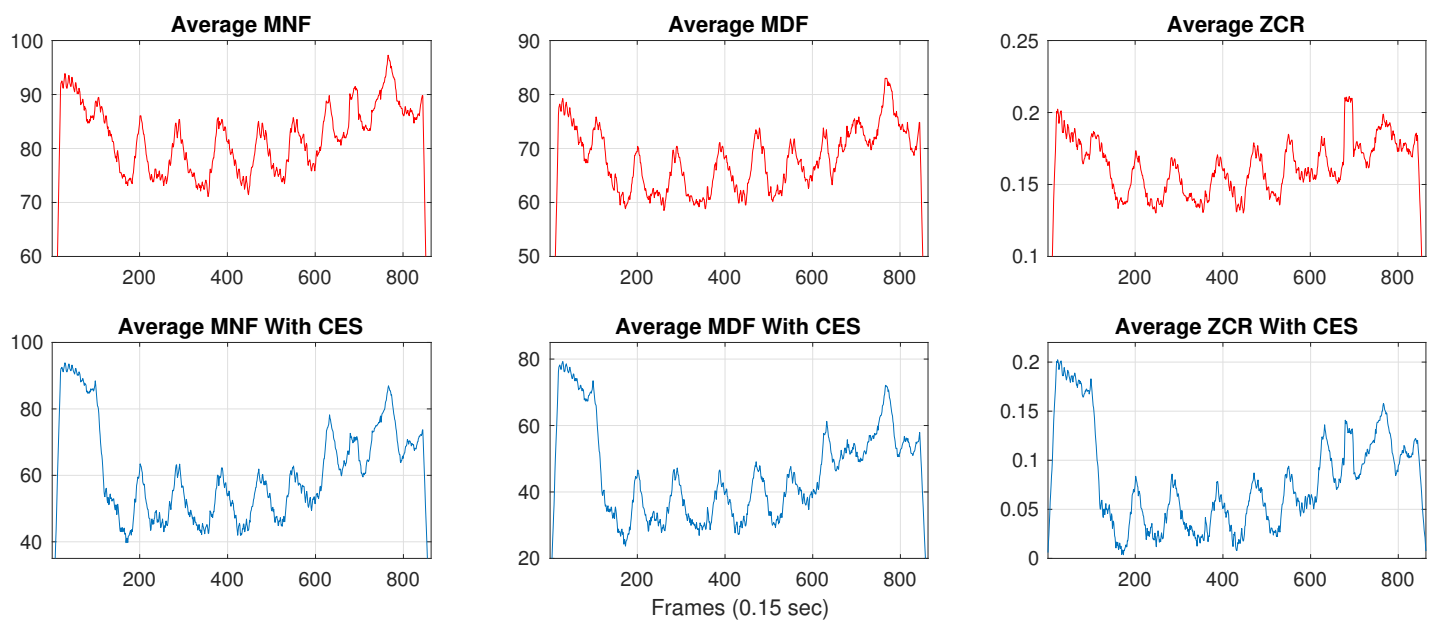

(a)
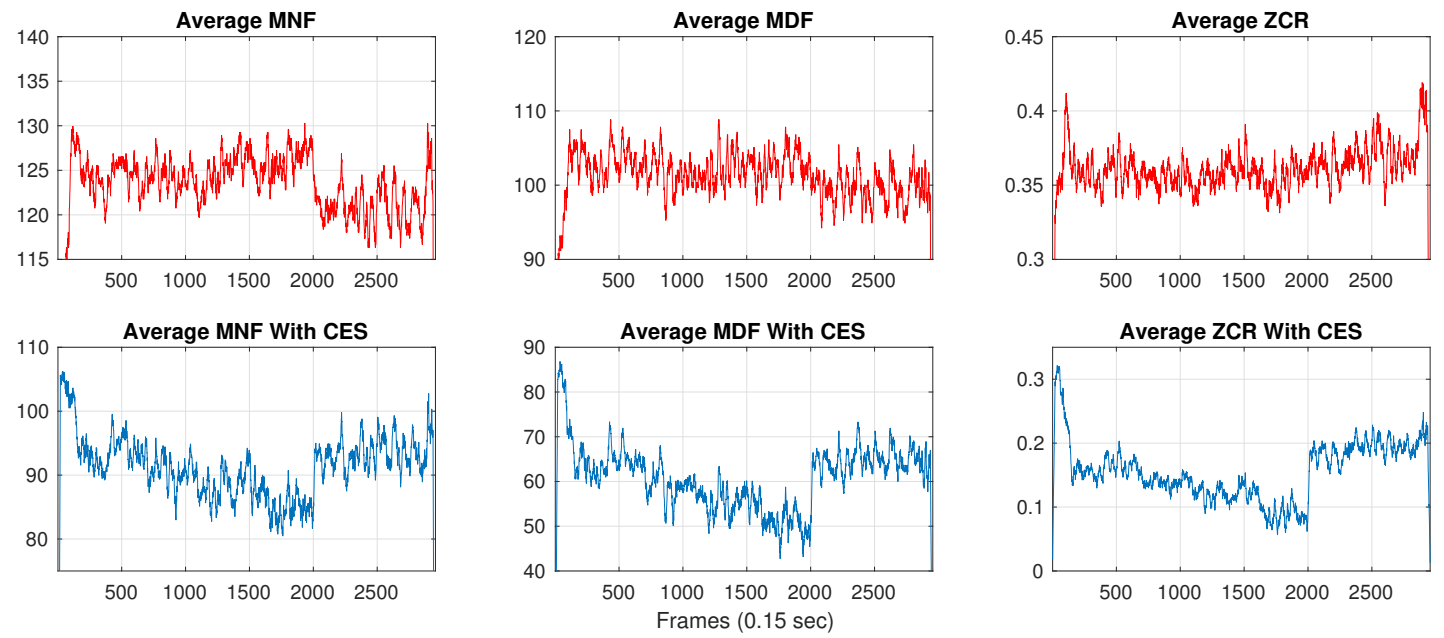

(b)

Figure 1. (a) The plots of averaged raw (top) and CES-applied (bottom) versions of MNF, MDF and ZCR values for the anaerobic exercises (b) The plots of averaged raw (top) and CES-applied (bottom) versions of MNF, MDF and ZCR values for the aerobic exercises

For the anaerobic exercise, subjects sat on an isokinetic dynamometer and made 7 sets of biceps curl exercise with 30 seconds of rests between each set. A set involved 10 reps of consecutive biceps curls with one's maximum possible effort. After the last set, each subject rested for 10 minutes, where subjects were asked to perform random contractions in order to monitor the recovery phase of their muscles during relaxation. In aerobic exercises subjects performed 4 sets of running (each 3 minutes) at speeds of 7, $8,9 \mathrm{~km} / \mathrm{h}$, respectively, and then 3 sets of walking (each 3 minutes) with a constant speed of $3 \mathrm{~km} / \mathrm{h}$. After each set, the subjects relaxed and rested for a minute. After the last rest, the subjects performed random calf contractions. However, EMG data alone is not sufficient for the objective evaluation of our algorithm. Therefore, we measured the blood lactate level using a lactate analyzer called LactateScout + at specific time intervals. The lactate level is measured from a blood sample gathered from the earlobe. For anaerobic exercises, we used the average peak torque values gathered from the isokinetic dynamometer.

\section{Comparisons of $\kappa$ Metrics With CES}

To see the advantages of CES algorithm, fatigue tracking metrics of MDF, MNF and ZCR (used as a $\kappa$ metric) are evaluated first alone and then with the use of CES for both aerobic and anaerobic exercises. The results are shown in the following subsections. Since 10 different subjects were involved in the experiments, the results are averaged. In the first part, subjects showed high effort involving anaerobic muscle activity, biceps curls, until the $7^{\text {th }}$ minute (i.e., $500^{\text {th }}$ frame), and then rested afterwards. After the intense anaerobic practice, namely $7^{\text {th }}$ minute, subjects continued giving physiological information through various muscle contractions, in order for us to track their recovery rate, since both the isokinetic dynamometer and sEMG analysis requires muscle activation for 

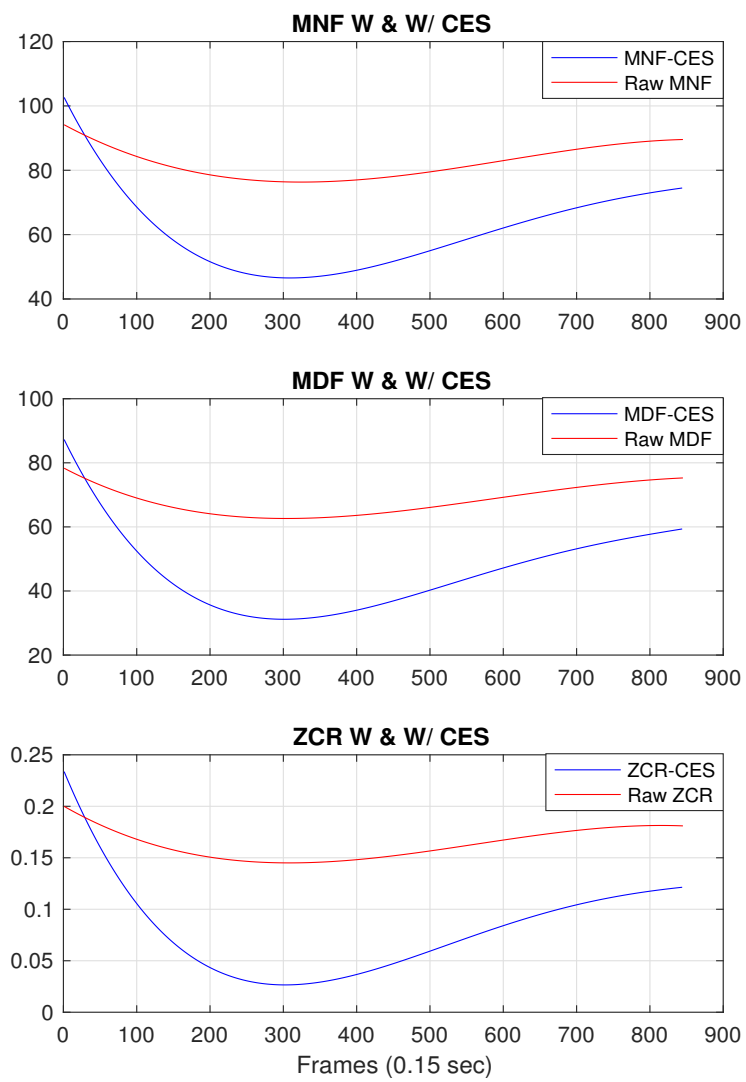

(a)
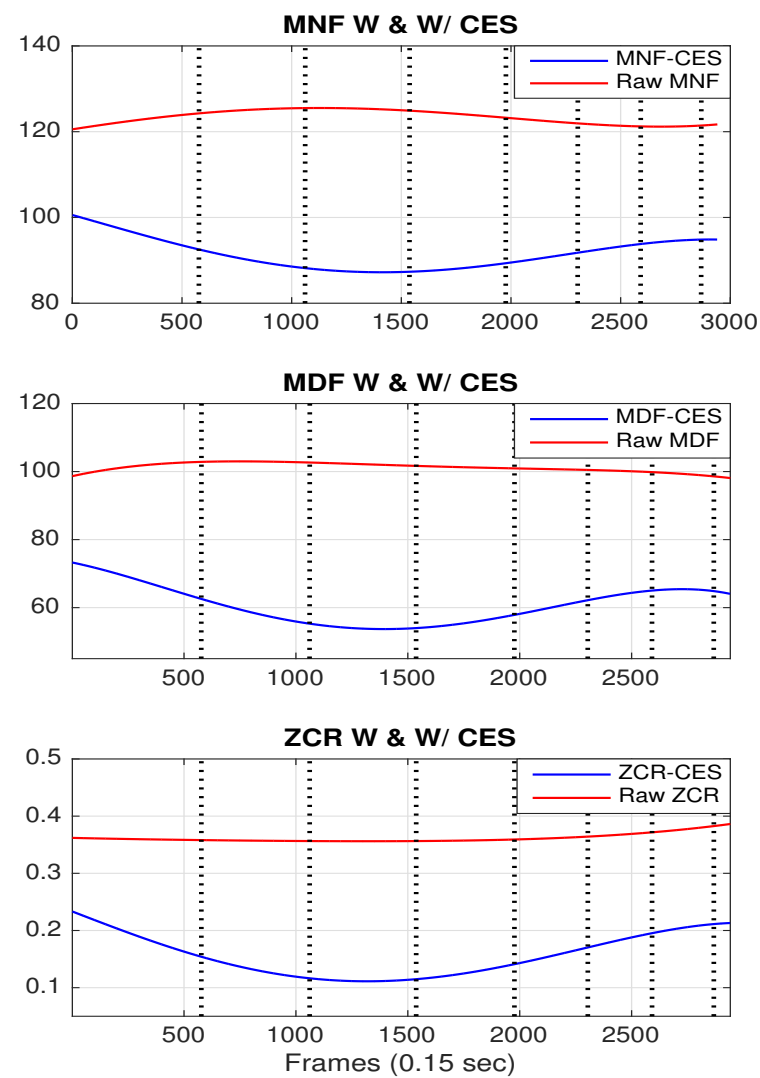

(b)

Figure 2. (a) The polynomially-fitted plots of CES-applied and raw versions of MNF (top), MDF (center) and ZCR (bottom) values for the anaerobic exercise. (b) The polynomially-fitted plots of CES-applied and raw versions of MNF (top), MDF (center) and ZCR (bottom) values for the aerobic exercises

further evaluation. The average and smoothed plots of raw and CES-applied $\kappa$ metrics are demonstrated in Figure $2 \mathrm{a}$ and $1 \mathrm{a}$. CES-applied results only demonstrate a slightly better classification between fatigued and non-fatigued stages. The reason of such small improvement of CES algorithm in this exercise is due to the little difference between contraction percentages, where each muscle activation showed approximately $2 V_{p-p}$ amplitude. In the second part of the experiments, EMG data involving aerobic exercises of several runs and walks, was analyzed. The average of selected $\kappa$ metrics are visualized in the top plots of Figure 1b, where it can be seen that the magnitude of the amplitude affects the $\kappa$ metric results and also the fatigue analysis. On the other hand, when CES algorithm is applied the effect of amplitude change is suppressed and thus a cleaner fatigue result is obtained. In order to obtain a smoother visualization, we have polynomially fitted the average calculations of resulting values in Figure 1b, as shown in Figure $2 b$. The smoothed (i.e. polynomially fitted) versions of the results demonstrate how the amplitude change affects the MNF, MDF and ZCR calculations. In this exercise, the average EMG power increases until the $15^{\text {th }}$ minute $\left(2000^{\text {th }}\right.$ contraction frame), since subjects gradually increase the running speed, and then drop to a smaller value for the rest of the activity due to the relaxation process, where the speed is constant and low. If Figure $2 \mathrm{~b}$ and $1 \mathrm{~b}$ are observed, raw versions of $\kappa$ increase until the $2000^{t h}$ contraction frame and then start to decrease afterwards. However, the results should have been the opposite, since the subjects make more effort (increasing fatigue) during the first 2000 frames and relax (decreasing fatigue) afterwards. The reason for this is the growing EMG RMS. That is to say, the EMG RMS increases with the increasing effort (i.e., higher speeds of running) and this shifts the spectrum to higher boundaries, while fatiguing situations tend to shift the frequency to lower bounds. Thus, it is uneasy to dynamically track fatigue states with the raw applications of $\kappa$ metrics. On the other hand, CES algorithm properly rejects the effect of EMG RMS. In the bottom plots of the Figure $2 \mathrm{~b}$ and $1 \mathrm{~b}$, it is seen that the resulting values are compatible with the exercise and for each $\kappa$ metric. Moreover, all the CES results ended up having the same waveform, whilst being different in their raw versions. 

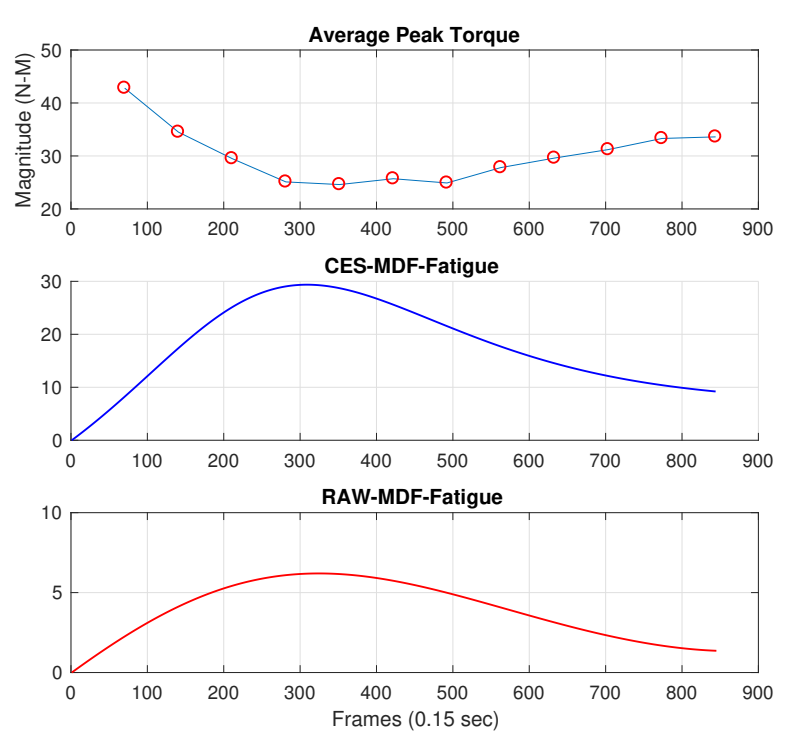

(a)
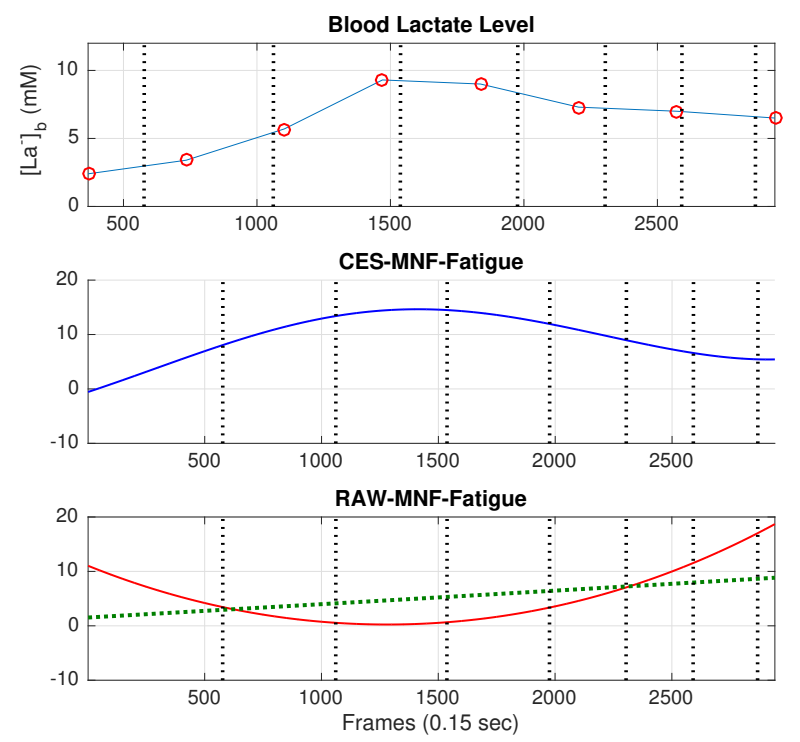

(b)

Figure 3. (a) The plots of average peak torque (top), CES-applied fatigue estimation (center), raw fatigue estimation (bottom) for the anaerobic exercises (b) The plots of lactate in blood (top), CES-applied fatigue estimation (center), raw fatigue estimation (bottom) for the aerobic exercises

\section{Comparisons of Anaerobic Exercise Results with the Isokinetic Dynamometer Results}

In this section, the isokinetic dynamometer results are used as an objective observation, besides sEMG-based analysis, for tracking biceps muscle fatigue level in order to properly compare CES's performance gains. The isokinetic dynamometer returned us the values of average peak torque, which decreases during muscle exhaustion and increases during muscle recovery. As predicted, in the first 500 contraction frames, the muscles get tired and thus the average peak torque value shows a drop from $42 \mathrm{~N}-\mathrm{M}$ to $24.6 \mathrm{~N}-\mathrm{M}$. Afterwards, during the recovery stage, the average peak torque increases from $24.6 \mathrm{~N}-\mathrm{M}$ to $35.4 \mathrm{~N}-\mathrm{M}$. The fatigue estimation through the sEMG-based analysis visualizes similar results with true exhaustion and recovery stage estimations. However, CES-applied $\kappa$ metrics outperforms its raw versions in creating better classification between fatigue and non-fatigue stages, which shows a higher similarity to isokinetic dynamometer results.

\section{Comparisons of Aerobic Exercise Results with the Lac- tate Test Results}

In this part, we compare the performance of our proposed method via an objective reference experiment, which is lactate test. Even though the fatigue value is predicted by simply likening it to the rate of effort extracted, it is still required to physiologically demonstrate the actual fatigue behavior. Lactate level in blood is an indicator, which is highly used to track human fatigue in both academic and medical fields. Thus, during the experiments we have also observed the lactate in blood and compare the average results with one of our $\kappa$ metrics, MNF, alone and with the use of CES algorithm. In the Figure 3b, the top plot shows the lactate in blood, which is continued by fatigue extracted with MNF (the bottom plot) and MNF with our proposed CES algorithm (the center plot). From the plots it can be clearly seen that our method extracts the fatigue, which highly resembles the lactate plot. All the changes in the lactate plot behavior are consistent with the results of MNF with CES. However, the fatigue plot that is acquired from the raw version of MNF, is not accurate and unable to detect the dynamic changes in fatigue. It can only evaluate the overall physiological state, when the values are linearly fitted (the green dotted plot). The reason is, as stated in Section 5.2, due to the effect of contraction amplitude on the spectral waveform. When the EMG signal amplitude increases because of the increase in exerted muscle force, it shifts the cumulated frequency of the acquired signal into higher bounds, thus creating a noise in fatigue analysis.

\section{CONCLUSION}

In this paper, we proposed a successful implementation of an algorithm to detect and surpass the effect of EMG signal power on its spectral analysis. Thus, the presented tool improved the performance of the EMG-based fatigue tracking methods of the state of the art. To begin with, we first classified the active and inactive EMG windows through an online adaptive thresholding method. After successfully differentiating the active EMG windows, we had them through varied fatigue tracking metrics with and without CES for both aerobic and anaerobic exercises. We compared the results through objective reference experiments, which are lactate (for aerobic activity) and isokinetic dynamometer (for anaerobic activity) tests. Results have shown us that the use of CES outperformed the raw use of fatigue tracking metrics. 


\section{REFERENCES}

1. biometricsltd. 2017. sEMG Sensor. http://www.biometricsltd.com/semg.htm. (2017).

2. A. Van Boxtel and L. R. B. Schomaker. 1983. Motor Unit Firing Rate During Static Contraction Indicated by the Surface EMG Power Spectrum. IEEE Transactions on Biomedical Engineering BME-30, 9 (Sept 1983), 601-609. DOI :

http://dx.doi.org/10.1109/TBME. 1983.325057

3. A. B. M. S. U. Doulah and M. A. Iqbal. 2012. An approach to identify myopathy disease using different signal processing features with comparison. In 2012 15th International Conference on Computer and Information Technology (ICCIT). 155-158. DOI:

http://dx.doi.org/10.1109/ICCITechn. 2012.6509759

4. M. Ergeneci, K. Gokcesu, E. Ertan, and P. Kosmas. 2018. An Embedded, Eight Channel, Noise Canceling, Wireless, Wearable sEMG Data Acquisition System With Adaptive Muscle Contraction Detection. IEEE Transactions on Biomedical Circuits and Systems 12, 1 (Feb 2018), 68-79. DOI:http://dx.doi.org/10.1109/TBCAS.2017.2757400

5. Y. Fan and Y. Yin. 2013. Active and Progressive Exoskeleton Rehabilitation Using Multisource Information Fusion From EMG and Force-Position EPP. IEEE Transactions on Biomedical Engineering 60, 12 (Dec 2013), 3314-3321. DOI : http://dx.doi.org/10.1109/TBME. 2013.2267741

6. E. Hazan, A. Agarwal, and S. Kale. 2007. Logarithmic regret algorithms for online convex optimization. Machine Learning 69, 2-3 (2007), 169-192.

7. E. Koutsos and P. Georgiou. 2014. An analogue instantaneous median frequency tracker for EMG fatigue monitoring. In 2014 IEEE International Symposium on Circuits and Systems (ISCAS). 1388-1391. DOI: http://dx.doi.org/10.1109/ISCAS.2014.6865403

8. Michael I. Lindinger and George J. F. Heigenhauser. 1991. The roles of ion fluxes in skeletal muscle fatigue. Canadian Journal of Physiology and Pharmacology 69, 2 (1991), 246-253. DOI : http://dx.doi.org/10.1139/y91-038 PMID: 2054741.

9. H. Liu. 2011. Exploring Human Hand Capabilities Into Embedded Multifingered Object Manipulation. IEEE
Transactions on Industrial Informatics 7, 3 (Aug 2011), 389-398. DOI :

http://dx. doi.org/10.1109/TII. 2011.2158838

10. M. M. Lowery and M. J. O'Malley. 2003. Analysis and Simulation of changes in EMG amplitude during high-level fatiguing contractions. IEEE Transactions on Biomedical Engineering 50, 9 (Sept 2003), 1052-1062. DOI : http://dx.doi.org/10.1109/TBME. 2003.816078

11. R. B. R. Manero, A. Shafti, B. Michael, J. Grewal, J. L. R. FernÃąndez, K. Althoefer, and M. J. Howard. 2016. Wearable embroidered muscle activity sensing device for the human upper leg. In 2016 38th Annual International Conference of the IEEE Engineering in Medicine and Biology Society (EMBC). 6062-6065. DOI : http://dx. doi.org/10.1109/EMBC. 2016.7592111

12. R. I. Pettigrew, W. J. Heetderks, C. A. Kelley, G. C. Y. Peng, S. H. Krosnick, L. B. Jakeman, K. D. Egan, and M. Marge. 2017. Epidural Spinal Stimulation to Improve Bladder, Bowel, and Sexual Function in Individuals With Spinal Cord Injuries: A Framework for Clinical Research. IEEE Transactions on Biomedical Engineering 64, 2 (Feb 2017), 253-262. DOI :

http://dx.doi .org/10.1109/TBME. 2016.2637301

13. C. U. Ranniger and D. L. Akin. 1997. EMG mean power frequency determination using wavelet analysis. In Engineering in Medicine and Biology Society, 1997. Proceedings of the 19th Annual International Conference of the IEEE, Vol. 4. 1589-1592 vol.4. DOI: http://dx.doi.org/10.1109/IEMBS. 1997.757017

14. SENIAM. 2017. Sensor Placement. http://www. seniam.org/bicepsbrachii.html. (2017).

15. F. B. Stulen and C. J. De Luca. 1981. Frequency Parameters of the Myoelectric Signal as a Measure of Muscle Conduction Velocity. IEEE Transactions on Biomedical Engineering BME-28, 7 (July 1981), 515-523. DOI :

http://dx.doi .org/10.1109/TBME. 1981. 324738

16. M. Yochum, T. Bakir, R. Lepers, and S. Binczak. 2012. Estimation of Muscular Fatigue Under Electromyostimulation Using CWT. IEEE Transactions on Biomedical Engineering 59, 12 (Dec 2012), 3372-3378. DOI : http://dx.doi.org/10.1109/TBME.2012.2215031 